\title{
Laser ablation-based one-step generation and bio-functionalization of gold nanoparticles conjugated with aptamers
}

\author{
Johanna G Walter', Svea Petersen², Frank Stahl' ${ }^{1}$, Thomas Scheper ${ }^{1}$, Stephan Barcikowski
}

\begin{abstract}
Background: Bio-conjugated nanoparticles are important analytical tools with emerging biological and medical applications. In this context, in situ conjugation of nanoparticles with biomolecules via laser ablation in an aqueous media is a highly promising one-step method for the production of functional nanoparticles resulting in highly efficient conjugation. Increased yields are required, particularly considering the conjugation of cost-intensive biomolecules like RNA aptamers.

Results: Using a DNA aptamer directed against streptavidin, in situ conjugation results in nanoparticles with diameters of approximately $9 \mathrm{~nm}$ exhibiting a high aptamer surface density (98 aptamers per nanoparticle) and a maximal conjugation efficiency of $40.3 \%$. We have demonstrated the functionality of the aptamer-conjugated nanoparticles using three independent analytical methods, including an agglomeration-based colorimetric assay, and solid-phase assays proving high aptamer activity. To demonstrate the general applicability of the in situ conjugation of gold nanoparticles with aptamers, we have transferred the method to an RNA aptamer directed against prostate-specific membrane antigen (PSMA). Successful detection of PSMA in human prostate cancer tissue was achieved utilizing tissue microarrays.

Conclusions: In comparison to the conventional generation of bio-conjugated gold nanoparticles using chemical synthesis and subsequent bio-functionalization, the laser-ablation-based in situ conjugation is a rapid, one-step production method. Due to high conjugation efficiency and productivity, in situ conjugation can be easily used for high throughput generation of gold nanoparticles conjugated with valuable biomolecules like aptamers.
\end{abstract}

\section{Background}

Gold nanoparticles (AuNPs) feature unique optical properties, including high surface plasmon resonance (SPR), enhanced absorbance and scattering with high quantum efficiency. In addition to their resistance against photobleaching, AuNPs perfectly fulfill requirements for use as colorimetric sensors and markers. For sensing or labeling of DNA targets, AuNPs can fairly easily be functionalized with DNA via thiol linkers, resulting in a highly ordered, self-assembled monolayer (SAM) [1,2]. Numerous colorimetric applications of DNA-conjugated AuNPs have already been developed[3].

\footnotetext{
* Correspondence: s.barcikowski@lzh.de

${ }^{2}$ Laser Zentrum Hannover, Hollerithallee 8, 30419 Hannover, Germany
}

Full list of author information is available at the end of the article
More recently, several applications of aptamer-conjugated AuNPs have been reported[4,5]. Aptamers are short, single-stranded DNA or RNA molecules that exhibit high specificity and affinity towards their corresponding target. Thus, aptamers can be thought of as nucleic acid analogues to antibodies that can be selected in vitro via SELEX (systematic evolution of ligands by exponential enrichment) against virtually any molecule, including proteins as well as small molecules like metal ions [6-8]. Aptamer-conjugated AuNPs have already been successfully used for the detection of proteins in a dry-reagent strip biosensor, [9] for detection of thrombin on surfaces, [4] for colorimetric detection of platelet-derived growth factor, [5] for detection of adenosine and potassium ions in an agglomeration-based approach, [10] for detection of thrombin in a dot blot assay [11] and for targeting and therapy of cancerous cells $[12,13]$. 
All applications of aptamer-conjugated AuNPs published so far have been based on chemical synthesis of AuNPs in the presence of reducing and stabilizing agents, and subsequent (ex situ) ligand exchange with aptamers. This ligand exchange might require heating and buffering in order to achieve satisfactory yields and surface coverage. The latter might be limited by interference from remaining reducing agents with the aptamer during the replacement process. Additionally, remaining precursors and/or reducing agents might result in a possible restriction of AuNPs use in biomedical applications $[14,15]$.

Recently, laser ablation of gold in a liquid environment has been used for the production of AuNPs $[16,17]$, using surfactants for growth quenching, resulting in narrow nanoparticle size distributions[18]. The advantages of laser-generated AuNPs include high purity in combination with unique surface characteristics. The $\mathrm{Au}$ surface of laser-generated AuNPs is partially oxidized, resulting in electrostatic stabilization of the colloid without the need for chemical additives. These partially positively-charged AuNPs, acting as electron acceptors, can interact directly with electron donors like amino or thiol groups in the ablation medium $[19,20]$. During laser ablation, the DNA acts as a capping agent, allowing precise size control of the resulting AuNPs, as it has been previously reported for the addition of cyclodextrines, biopolymers, etc[21]. Recently, a direct comparison of conventional ex situ conjugation of lasergenerated AuNPs and laser-ablation-based in situ conjugation of AuNPs with DNA has revealed a four times higher conjugation efficiency when using the laser-ablation-based procedure. In comparison to AuNPs produced by chemical synthesis and subsequent ex situ conjugation, AuNPs generated using laser-ablationbased in situ conjugation exhibit up to five times higher surface coverage[22]. Hence, bio-conjugation during laser ablation presents a rapid and efficient preparation method, especially for the conjugation of valuable biomolecules like aptamers or vectors. The high surface coverage of DNA-modified AuNPs produced by in situ conjugation may be especially advantageous for applications including cellular uptake of AuNPs. In this context, Giljohann et al. have found that the extent of cellular uptake of DNA-modified AuNPs can be increased by enhancing the DNA loading[23]. Moreover, high DNA densities can also facilitate cooperative binding, resulting in increased association constants with a given target, e.g. in intracellular gene regulation[24]. Another important parameter that can be modulated via surface coverage is the immune response induced by DNA-modified AuNPs. Higher DNA densities efficiently limit the immune response as measured by Interferon- $\beta$ expression in mouse macrophages[25].
In spite of these benefits, the use of laser-ablationbased in situ conjugation for the generation of aptamerconjugated AuNPs has not yet been reported.

We show the functionalization of nanoparticles with aptamers during femtosecond-pulsed, laser-induced gold nanoparticle formation in an aqueous media using a DNA aptamer directed against streptavidin as a model system. In order to demonstrate the applicability of aptamer-conjugated AuNPs generated via laser ablation in complex biomedical applications, we have used an RNA aptamer directed against prostate-specific membrane antigen (PSMA) for the detection of PSMA in human prostate cancer tissue utilizing tissue microarrays.

\section{Results and Discussion}

\section{Choice of aptamer orientation and spacer design}

In order to ensure aptamer activity, several factors concerning the ability of the aptamer to fold into the correct three-dimensional structure have been considered. We have previously reported the application of an aptamer directed against streptavidin (referred to as miniStrep) in a protein microarray format[26,27]. Using this approach, we have found that the miniStrep aptamer requires an additional spacer placed between the aptamer and the substrate to show activity that is slightly higher when immobilized via its 3 ' terminus. Thus, we decided to use 3' orientation. An additional oligothymidine (T10) spacer was placed between the disulfide group and the aptamer sequence. Tymidine was chosen, since this nucleotide has the lowest affinity towards the gold surface[28]. Thus, nonspecific binding of the spacer bases to gold is minimized, which should increase the surface loading and improve elevation of the aptamer away from the nanoparticle surface. Taking these considerations into account, the miniStrep aptamer construct used in this work was the following: TCT GTG AGA CGA CGC ACC GGT CGC AGG TTT TGT CTC ACA G - $\mathrm{T}_{10}-\left(\mathrm{CH}_{2}\right)_{3}-\mathrm{S}-\mathrm{S}-\left(\mathrm{CH}_{2}\right)_{6} \mathrm{OH}$.

We decided to immobilize the anti-PSMA aptamer via the 3'terminus. According to Lupold et al., the aptamer can be subjected to 3 ' truncation of up to 15 nucleotides without losing its affinity to PSMA[29]. Since the 3' terminal bases are not necessary for target recognition, we decided to omit the use of an additional oligonucleotide spacer. Instead, hexaethylenglycol was chosen as a spacer, because it does not exhibit intermolecular repulsion, which is one cause of low DNA loading on AuNPs [30]. Furthermore, it only occupies a small surface area, which allows high packing densities,[30] and is known to minimize nonspecific protein binding[31]. Therefore, the aptamer construct used was the following: GGG AGG ACG AUG CGG AUC AGC CAU GUU UAC GUC ACU CCU UGU CAA UCC UCA UCG GCA 


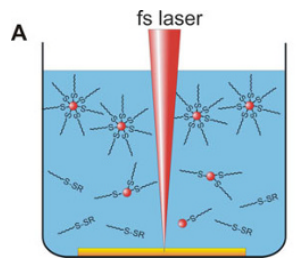

B
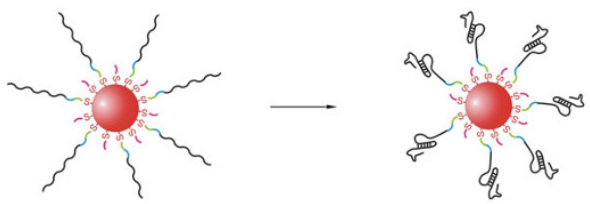

TCT GTG AGA CGA CGC ACC GGT CGC AGG TTT TGT CTC ACA G TTTTTTTTTTT $\left(\mathrm{CH}_{2}\right)_{3}-\mathrm{S}-\mathrm{S}-\left(\mathrm{CH}_{2}\right)_{6} \mathrm{OH}$

Figure 1 Generation of aptamer-conjugated AuNPs via in situ conjugation. (A) Schematic illustration of in situ conjugation of AuNPs with aptamers during laser ablation in an aqueous aptamer solution. (B) Spacer design and resulting mixed monolayer conjugated nanoparticles. Mixed monolayer formation and careful spacer design contribute to correct aptamer folding.

GAC GAC UCG CCC GA- $\left(\mathrm{CH}_{2} \mathrm{CH}_{2} \mathrm{O}\right)_{6}-\left(\mathrm{CH}_{2}\right)_{6}$-S-S$\left(\mathrm{CH}_{2}\right)_{6} \mathrm{OH}$.

The aptamers were directly used in the laser ablation process without prior dithiothreitol (DTT) treatment (Figure 1A). According to Dougan et al., this does not affect surface coverage[32]. Moreover, the mercaptohexanol $(\mathrm{MCH})$ of the mixed disulfide (aptamer-S-S- $\left(\mathrm{CH}_{2}\right)$ $\left.{ }_{6} \mathrm{OH}\right)$ may serve as a co-adsorbent, eliminating unspecific binding to the gold surface, by occupying free binding sites[33]. Due to the formation of a mixed monolayer consisting of aptamers and short organic residues, the available space for optimal aptamer folding is enhanced (Figure 1B).

\section{In situ conjugation}

Due to rapid, one-step processing, laser-ablation-based in situ conjugation enables fast screening of different conjugation conditions. Utilizing this high throughput potential, we have determined optimal conjugation conditions by using different concentrations of miniStrep aptamer in a Tris(hydroxymethyl)-aminomethan (Tris) buffer during laser ablation. Per investigated concentration, the laser ablation process took less than two minutes. A UV/VIS spectrum of AuNPs produced via laser

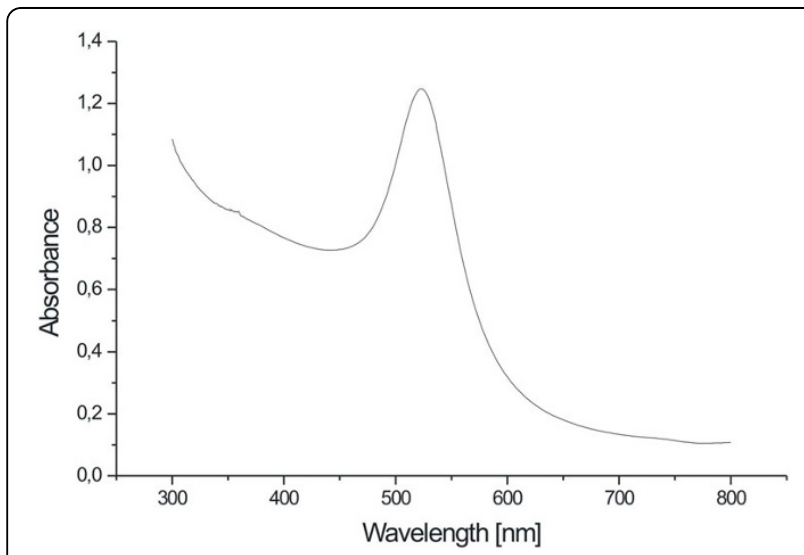

Figure 2 UV/VIS spectrum of aptamer-conjugated AuNPs. The spectrum was obtained with an as prepared AuNP solution after in situ conjugation with $5 \mu \mathrm{M}$ anti-streptavidin aptamer. ablation in the presence of $5 \mu \mathrm{M}$ aptamer can be found in Figure 2.

DLS measurements demonstrate that the hydrodynamic diameter $\left(d_{h}\right)$ of the AuNPs increases with increasing aptamer concentrations (Figure $3 \mathrm{~A}$ ). While $d_{h}$ after ablation in Tris buffer (without aptamer) is $7 \mathrm{~nm}$, $\mathrm{d}_{\mathrm{h}}$ increases with increasing aptamer concentrations up to $5 \mu \mathrm{M}$, and finally reaches a plateau of approximately $60-70 \mathrm{~nm}$ (Figure 3A). We assume that this $d_{h}$ increase is a result of cumulative aptamer loading on the gold surface. At low surface coverage, the aptamer lays flat on the surface, due to non-specific binding via the lone

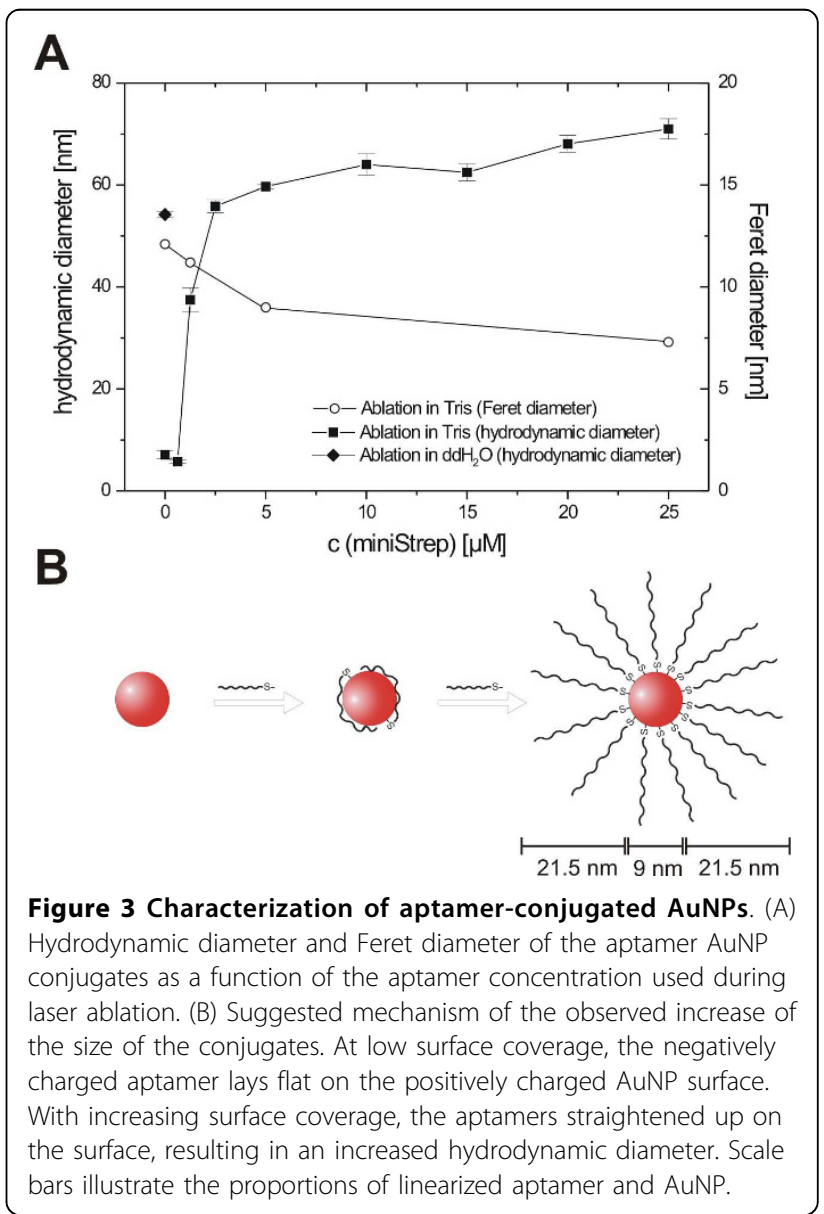


nitrogen electron pairs of the nucleotides. As the surface coverage increases, the aptamers are forced to adopt a more perpendicular conformation, due to electrostatic repulsion of the aptamers' negatively charged phosphate backbones, resulting in a $d_{h}$ increase (Figure $3 B$ ). We estimated the length of the aptamer (including $\mathrm{T}_{10}$ spacer) to be $21.5 \mathrm{~nm}$, using a base to base distance for ssDNA of $0.43 \mathrm{~nm}[34]$. For an aptamer-conjugated AuNP of $9 \mathrm{~nm}$ core size, this results in a diameter of approximately $52 \mathrm{~nm}$, which is close to the observed plateau of $d_{h}$, and supports our assumption (Figure 3B).

On first sight, the AuNPs hydrodynamic diameter increase seems to be contradictory to our previous finding of a growth quenching effect induced by increasing DNA concentrations[19]. But in contrast to our previous work, here the ablation was performed in Tris buffer. Tris interacts with the surface of the embryonic AuNPs, resulting in prevention of further post-ablation nanoparticle agglomeration. Consequently, the AuNPs produced in Tris buffer are already stabilized by the buffer molecule, resulting in reduced diameters $\left(d_{h}=7.1 \pm 0.8 \mathrm{~nm}\right.$ in Tris buffer versus $54.2 \pm 0.6 \mathrm{~nm}$ in $\mathrm{dd}_{2} \mathrm{O}$ (Figure 3A)) and a diminished influence of the oligonucleotide concentration on the nanoparticle size. This assumption is supported by TEM analysis data. The Feret diameter $\left(\mathrm{d}_{\text {Feret }}\right)$ of AuNPs produced in Tris buffer slightly decreases from 12.1 to $7.3 \mathrm{~nm}$ with increasing aptamer concentration (Figure 3A). In comparison to the Tris molecule, the thiolated aptamer exhibits a higher affinity towards the gold surface, resulting in better stabilization of embryonic particles, and thus smaller AuNPs, as detected via TEM analysis. Although there may be some portion of Tris-aptamer ligand exchange after nanoparticle generation, the size quenching effect observed by TEM analysis confirms successful in situ bio-conjugation during laser ablation.

In addition to the AuNP size, we have determined aptamer loading (Table 1). For AuNPs produced by laser ablation in a $5 \mu \mathrm{M}$ aptamer solution (in situ), we found a loading of 98 aptamers per nanoparticle, corresponding to $65 \mathrm{pmol} / \mathrm{cm}^{2}$. This aptamer loading is higher than the results achieved by post production (ex situ) modification of chemically synthesized AuNPs with short oligonucleotides (Demers et al:: $34 \mathrm{pmol} / \mathrm{cm}^{2}$ ),[35] and the aptamer loading to chemically synthesized AuNPs reported by Huang et al. $\left(13 \mathrm{pmol} / \mathrm{cm}^{2}\right)$ [5]. The high aptamer loading achieved by in situ conjugation confirms high availability of laser-generated AuNPs for bio-conjugation. The conjugation efficiency was calculated as the portion of provided aptamer bound to the nanoparticle surface (Table 1). At a $1.25 \mu \mathrm{M}$ aptamer concentration, $40.3 \%$ of the available aptamer binds to the AuNPs, which demonstrates the suitability of the method for efficient conjugation of valuable biomolecules. Since we have observed no denaturation of the aptamer during laser ablation (as discussed in the next section), the remaining aptamer can be reused.

After the ablation process, conjugates were slowly transferred into the aptamer selection buffer by adding $\mathrm{NaCl}$ and $\mathrm{MgCl}_{2}$. During this salting process, we observed precipitation of AuNPs produced at aptamer concentrations lower than $5 \mu \mathrm{M}$. The higher stability of AuNPs conjugated at aptamer concentrations of $5 \mu \mathrm{M}$ (or higher) coincides with the plateau in the hydrodynamic diameter (Figure 3B), and indicates better stabilization due to higher surface coverage.

All further experiments were performed with AuNPs produced in a $5 \mu \mathrm{M}$ aptamer solution, which was a compromise between maximal aptamer density and minimal aptamer consumption (Table 1 ). Under these conditions, we could produce $75 \mu \mathrm{g}(150 \mu \mathrm{g} / \mathrm{ml}) \mathrm{miniS}$ trep-conjugated AuNPs in less than two minutes. The free aptamer was removed by centrifugation. In order to maintain conjugate activity, centrifugation was performed under rather mild conditions $(16600 \times \mathrm{g})$. This procedure results in a slightly increased average Feret diameter $(14.6 \mathrm{~nm})$ due to loss of small nanoparticles (Figure 4).

It should be noted that the high immobilization efficiency, and thus high aptamer consumption, results in decreasing aptamer concentrations during the laser ablation process. As we suppose that the aptamer conjugation takes place in the millisecond to second regime after the collapse of the cavitation bubble, the aptamer loading of NPs will also decrease over this period of time. If more homogeneous aptamer loadings are

Table 1 Characterization of aptamer-conjugated gold nanoparticles

\begin{tabular}{|c|c|c|c|c|c|}
\hline $\begin{array}{l}\mathrm{C}_{(\text {miniStrep })} \\
{[\mu \mathrm{M}]}\end{array}$ & $\begin{array}{l}\mathrm{d}_{\mathrm{h}}(\mathrm{DLS})^{a} \\
{[\mathrm{~nm}]}\end{array}$ & $\begin{array}{l}\mathrm{d}_{\text {Feret }} b \\
{[\mathrm{~nm}]}\end{array}$ & Aptamer/AuNP & $\begin{array}{l}\text { Aptamer/A AuNP } \\
{\left[\mathrm{pmol} / \mathrm{cm}^{2}\right]}\end{array}$ & $\begin{array}{l}\mathrm{E}_{\text {con }}{ }^{d} \\
{[\%]}\end{array}$ \\
\hline 0 & $7.1 \pm 0.8$ & $12.1 \pm 9.8$ & - & - & - \\
\hline 1.25 & $37.5 \pm 2.3$ & $11.2 \pm 4.2$ & $80 \pm 2$ & $33.93 \pm 1.04$ & $40.3 \pm 0.3$ \\
\hline 5 & $61.1 \pm 1.9$ & $9.0 \pm 5.0$ & $98 \pm 4$ & $64.58 \pm 1.82$ & $19.8 \pm 0.7$ \\
\hline 25 & $71.0 \pm 2.0$ & $7.3 \pm 2.5$ & $74 \pm 11$ & $73.90 \pm 7.22$ & $6.1 \pm 0.9$ \\
\hline
\end{tabular}

Summary of data obtained for AuNPs conjugated with miniStrep aptamer in Tris buffer $\left({ }^{a}\right.$ Hydrodynamic diameter, ${ }^{b}$ Feret diameter, ${ }^{c}$ Aptamers per AuNP surface, ${ }^{d}$ Conjugation efficiency). 


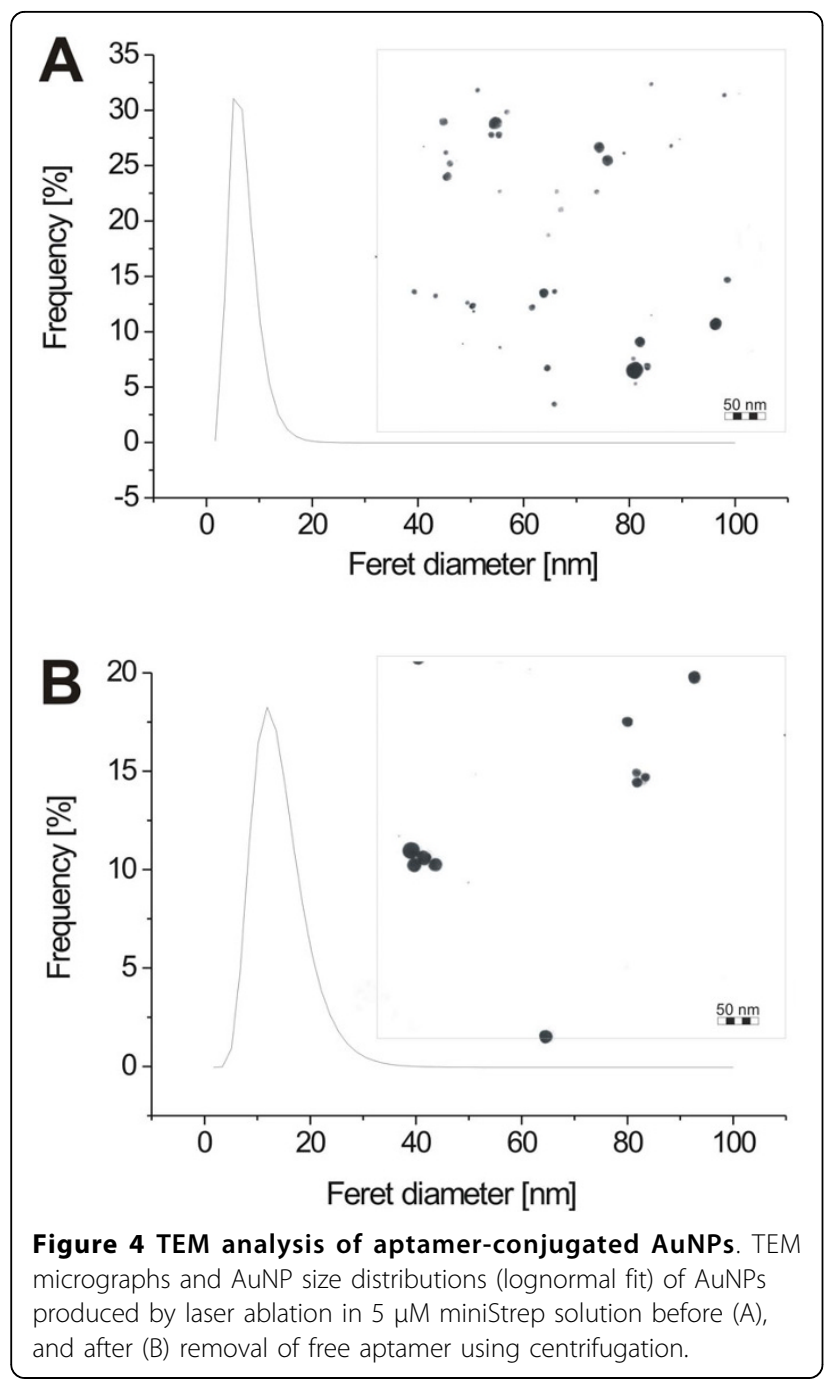

required, this can be achieved by applying higher aptamer concentrations and/or by shortening ablation time.

\section{Functionality of miniStrep-conjugated AuNPs}

Functionality of the immobilized miniStrep aptamer was confirmed by using three independent methods. First, a classical, agglomeration-based method was applied. A fixed amount of AuNPs (0.69 nM) conjugated with miniStrep aptamer was incubated with different amounts of streptavidin (0 - $15.9 \mathrm{nM})$, and UV/VIS was detected. Since streptavidin is a tetrameric protein, agglomeration can be observed as a red shift of $\mathrm{SPR}_{\mathrm{Max}}$ (Figure 5A). The shift in $\mathrm{SPR}_{\text {Max }}$ increases with increasing concentrations of streptavidin, and reaches a maximum at a streptavidin concentration of $2 \mathrm{nM}$. In addition to the $\mathrm{SPR}_{\text {Max }}$ shift, we observed the formation of a red film on the wall of the reaction vessel at streptavidin concentrations from $1 \mathrm{nM}$ to $4 \mathrm{nM}$. Simultaneously, we observed a loss of AuNPs in the solution. Based on absorbance at $380 \mathrm{~nm}$, the loss of particles was calculated to be $86.5 \%$. We assume that the red film is composed of large agglomerates, while small agglomerates stay in the solution and can be detected via the shift of $\mathrm{SPR}_{\mathrm{Max}}$ and TEM analysis. TEM micrographs of the agglomerates indicated a defined composition and tetrahedral structure of these agglomerates (Figure 6). Based on TEM analysis, we determined an agglomerate size of $35 \mathrm{~nm}$ (edge-to-edge length). In order to verify the proposed tetrahedral structure, we calculated the size of the agglomerates based on the observed shift of SPR $\mathrm{Max}_{\text {, }}$ utilizing the "plasmon ruler equation":[36]

$$
\frac{\Delta \lambda}{\lambda_{0}} \approx 0,18 \times \exp \left(\frac{-\left(\frac{s}{D}\right)}{0,23}\right)
$$

This approximation describes the dependency between the observed shift of $\mathrm{SPR}_{\text {Max }}(\Delta \lambda)$ on the interparticle gap (s) and nanoparticle size (D). Using our experimental results $\left(\Delta \lambda=6 \mathrm{~nm} ; \lambda 0=523.5 \mathrm{~nm} ; \mathrm{D}=\mathrm{d}_{\text {Feret }}=14.6\right.$ $\mathrm{nm}$ ), we calculated an interparticle distance of $9.3 \mathrm{~nm}$, and an edge-to-edge length of the proposed tetrahedron of $38.5 \mathrm{~nm}$. Taking into account that equation (1) is an empirical approximation established for a pair of interacting nanoparticles rather than for a tetramer, and considering that the geometry of streptavidin is not perfectly tetrahedral, the deviation of $10 \%$ between the agglomerate sizes measured by TEM analysis and calculated from the shift of $\mathrm{SPR}_{\mathrm{Max}}$ seems to be acceptable. Good agreement between the agglomeration sizes obtained using two independent methods supports the proposed tetrahedral structure of the agglomerates.

At streptavidin concentrations above $2 \mathrm{nM}$, the $\mathrm{SPR}_{\text {Max }}$ shift decreases, due to saturation of aptamers immobilized on the AuNPs surface with streptavidin (Figure 5B). This saturation effect is in accordance with the observations of Huang et al., who used an aptamer against a dimeric protein (platelet-derived growth factor)[5].

In order to gain quantitative insight into streptavidin binding and thus aptamer activity, the AuNPs were incubated with an excess of $\mathrm{Cy} 3$ labeled streptavidin. The agglomerates of aptamer-coated nanoparticles and attached streptavidin were removed using ultracentrifugation, and the amount of bound streptavidin was determined by measuring the remaining streptavidin concentration in the supernatant.

Since aptamer loading was determined for the whole AuNP population generated by laser ablation $\left(\mathrm{d}_{\mathrm{Feret}}=\right.$ $9.0 \mathrm{~nm}$ ), and the binding of Cy3 labeled streptavidin was performed with the AuNP subpopulation resulting from 


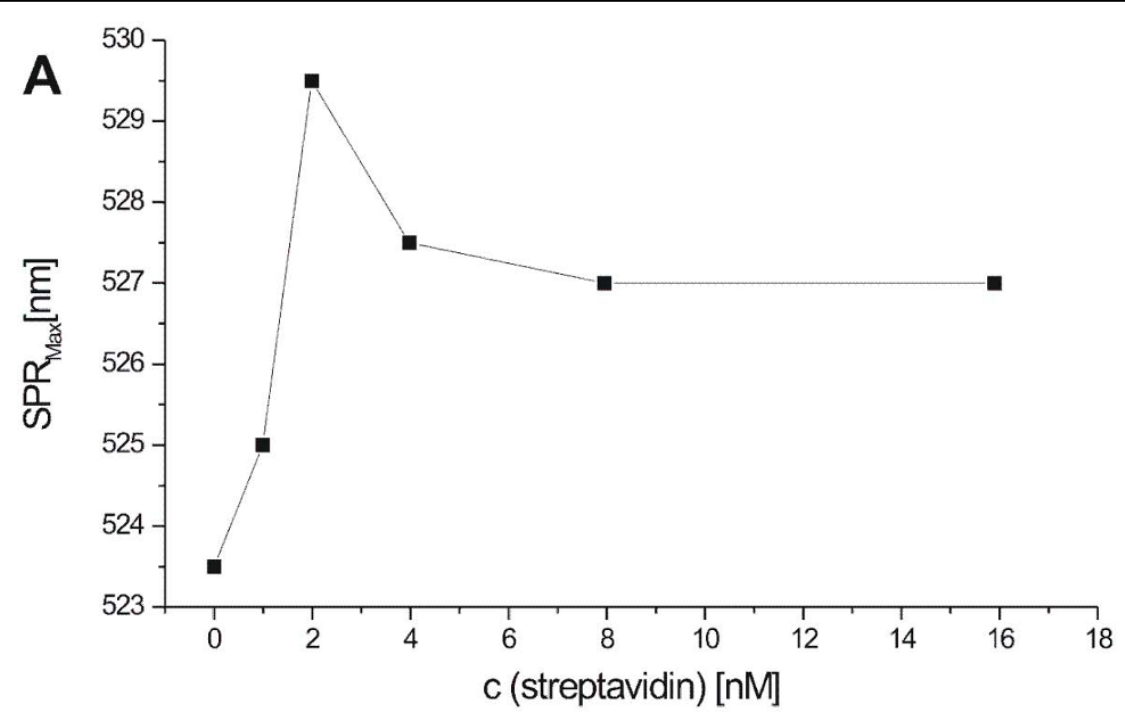

B
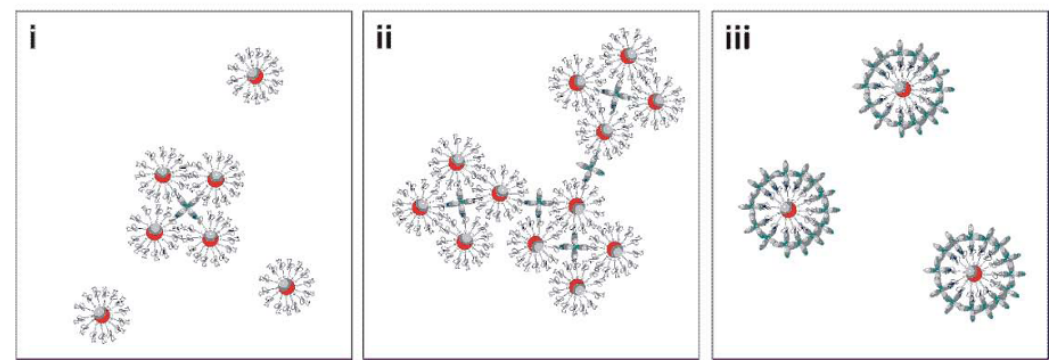

Figure 5 Verification of the activity of aptamer-conjugated AuNPs. (A) The shift of the SPR maximum clearly indicates the formation of agglomerates in the presence of streptavidin. (B) Schematic illustration of the formation of agglomerates as a function of streptavidin concentration: At low streptavidin concentrations, relatively small agglomerates occur (i). At medium streptavidin concentrations, the tetrameric protein induces the formation of large agglomerates (ii). An excess of streptavidin inhibits the formation of agglomerates by saturation of the aptamers bound to the nanoparticle surface (iii).

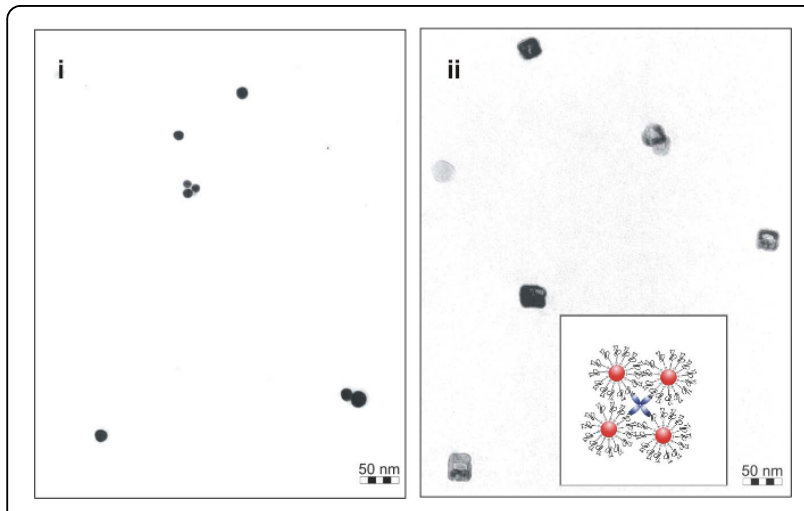

Figure 6 TEM analysis of AuNPs conjugated with aptamers against streptavidin. TEM micrographs of AuNPs without streptavidin (i), and after incubation with streptavidin (ii). The insert displays a scheme of the proposed composition of the agglomerates. Please note that the agglomerates are displayed in a simplified manner in the scheme, de facto streptavidin is not planar but tetrahedral. removal of the free aptamer by centrifugation $\left(\mathrm{d}_{\mathrm{Feret}}=\right.$ $14.6 \mathrm{~nm}$ ), it was not possible to compare aptamer loading directly to the amount of bound streptavidin. In order to estimate the aptamer activity, it was assumed that aptamer density $\left(\mathrm{pmol} / \mathrm{cm}^{2}\right)$ is not significantly affected by the nanoparticle diameter. Based on this assumption, the aptamer loading was calculated to be $64.58 \pm 1.82 \mathrm{pmol} / \mathrm{cm}^{2}$, and the amount of streptavidin bound to the AuNP surface was $67.23 \pm 0.76 \mathrm{pmol} / \mathrm{cm}^{2}$, resulting in approximately $100 \%$ aptamer activity. This indicates that the aptamer is not degraded during the laser ablation process, and optimal aptamer folding was achieved by careful design of the spacer.

To examine the applicability of the aptamer-conjugated AuNPs in solid phase assays, we performed a simple dot blot assay (Figure 7A). Within this assay, BSA was used as a negative control. BSA was chosen because it exhibits a mildly acidic isoelectric point (pI) similar to the $\mathrm{pI}$ of streptavidin (pI 5-6) resulting in a comparable 


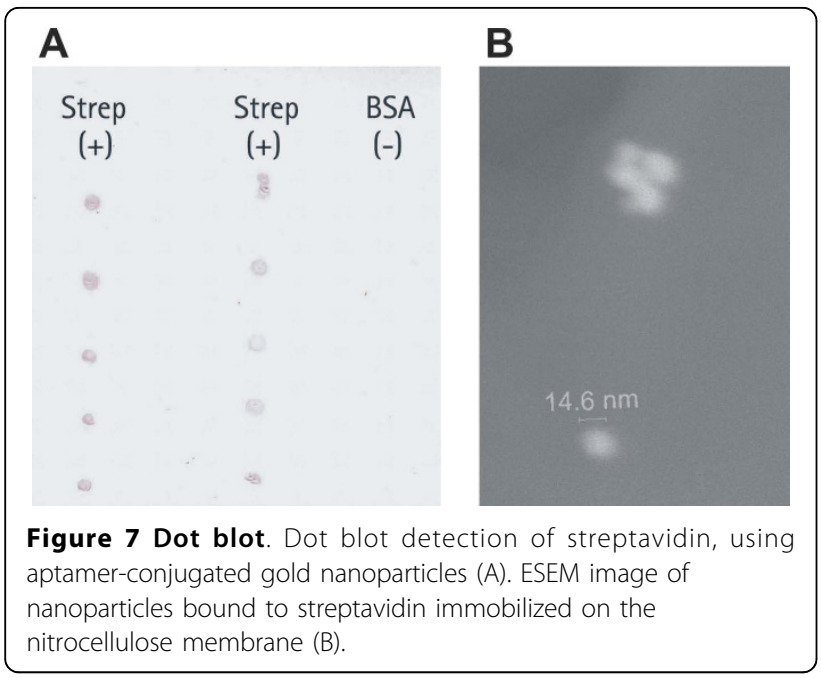

negative net charge of the two proteins under the given conditions ( $\mathrm{pH}$ 7.4). In order to exclude the possibility of electrostatic interactions between the negatively charged proteins and the positively charged AuNPs, the experiment was repeated with unconjugated nanoparticles. The nanoparticle aptamer conjugates bind only to the immobilized streptavidin, and no binding can be observed to BSA. Using unconjugated nanoparticles, no binding of AuNPs to the immobilized proteins occurred (data not shown). This clearly demonstrates the specific binding of AuNPs to streptavidin via the aptamer conjugated to the nanoparticle surface.

The AuNPs bound to streptavidin during the dot blot assay were further analyzed via ESEM. The ESEM micrograph affirms the Feret diameter of the nanoparticles determined by TEM (Figure 7B).

\section{Functionality of anti-PSMA-conjugated AuNPs}

Encouraged by the positive performance of the dot blot assay, our next aim was to prove the applicability of aptamer-conjugated AuNPs in more complex and demanding solid-phase assays. Therefore, we used AuNPs conjugated with an aptamer directed against PSMA for detection of PSMA in prostate cancer (adenocarcinoma) tissue sections.

AuNPs conjugated with anti-PSMA aptamer show a staining pattern similar to anti-PSMA antibody. In both cases, a positive staining of acinar epithelial cells was observed (Figure 8). In tissue sections treated with antiPSMA aptamer-conjugated AuNPs, an additional staining of muscle cells was observed that was not detected in the positive control. To ensure that the binding to PSMA is based on the affinity of the anti-PSMA aptamer rather than on electrostatic interaction between the target protein and the highly negatively charged aptamers, AuNPs conjugated with anti-streptavidin aptamers were used.

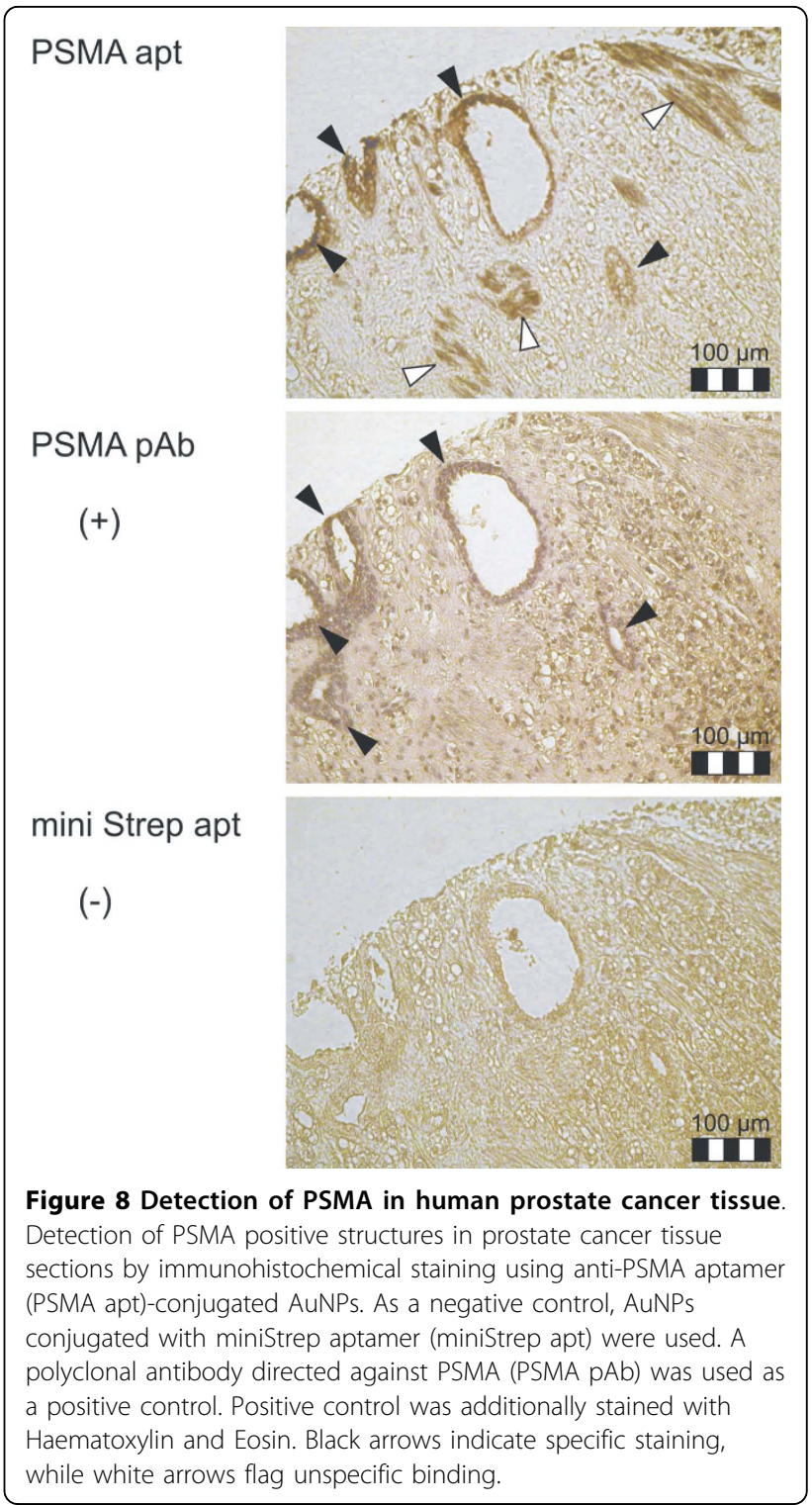

Since the negative control does not show positive binding to epithelial cells or false positive binding to muscle cells, we assume the binding of anti-PSMA conjugates to muscle cells to be induced by the specific three-dimensional structure of the anti-PSMA aptamer. A positive staining of smooth muscle cells in prostate cancer has also been reported for one monoclonal PSMA antibody (7E11),[37] and some authors assume that there may be a "PSMAlike" target in smooth muscle cells[38,39]. Following this consideration, the binding of AuNPs conjugated with anti-PSMA aptamer to muscle cells may be the result of cross-reactivity of the aptamer with this unknown "PSMA-like" target. In summary, our results demonstrate that the anti-PSMA aptamer AuNP conjugates can detect PSMA in acinar epithelial cells of human prostate cancer. This exemplifies the broad applicability of aptamer- 
conjugated AuNPs, even in highly complex biological matrices and bio-imaging applications.

\section{Conclusions}

We have demonstrated the suitability of laser-ablationbased in situ bio-conjugation for the production of functional, aptamer-conjugated gold nanoparticles. Exploiting the potential of this rapid, one-step method for high throughput screening, we have optimized the conjugation regarding aptamer loading and conjugation efficiency. To address the general applicability of the method, we have utilized two different aptamers composed of DNA and RNA. The high degree of aptamer activity determined on AuNP surface verifies that there is no heat-induced denaturation of the aptamer during laser ablation. We have proven the functionality of conjugates using three different methods (agglomeration-based assay, dot blot assay, tissue microarray), indicating the broad applicability of aptamer-conjugated gold nanoparticles for bio-analytical applications, even in highly demanding assays. Moreover, in situ conjugation avoids possible contamination by toxic educts, residual reducing agents or preservatives. Thus, this method could also be especially advantageous for use in medical applications.

Since in situ conjugation is a fast and simple one-step approach to generate pure conjugated AuNPs with high conjugation efficiency and productivity, it can be easily used for the high throughput production of large amounts of different conjugated nanoparticles. The higher conjugation efficiencies are beneficial for highpriced biomolecules, and the comparably high surface coverage is desirable for cellular uptake, which depends on the DNA density on the AuNPs surface. Moreover, such high surface densities may assist cooperative binding and may decrease the immune response against AuNPs.

\section{Methods \\ Materials}

All chemicals were purchased from Sigma-Aldrich (Steinheim, Germany) or Fluka Chemie AG (Taufkirchen, Germany), and used as received. The aptamer against streptavidin (TCT GTG AGA CGA CGC ACC GGT CGC AGG TTT TGT CTC ACA G - $\mathrm{T}_{10^{-}}\left(\mathrm{CH}_{2}\right)_{3^{-}}$ S-S- $\left(\mathrm{CH}_{2}\right)_{6} \mathrm{OH}$, referred to as miniStrep) [26] and antiPSMA aptamer (GGG AGG ACG AUG CGG AUC AGC CAU GUU UAC GUC ACU CCU UGU CAA UCC UCA UCG GCA GAC GAC UCG CCC GA$\left(\mathrm{CH}_{2} \mathrm{CH}_{2} \mathrm{O}\right)_{6}-\left(\mathrm{CH}_{2}\right)_{6}$-S-S- $\left.\left(\mathrm{CH}_{2}\right)_{6} \mathrm{OH}\right)$ [29] were purchased from Biospring GmbH (Frankfurt, Germany). The gold foil was $0.1 \mathrm{~mm}$ thick and had $>99.99 \%$ purity, and was obtained from Goodfellow GmbH (Bad Nauheim, Germany).

\section{Generation of aptamer-conjugated AuNPs}

Laser ablation was performed in the same buffer system the aptamer was originally selected in. For the miniStrep aptamer, $50 \mathrm{mM}$ Tris(hydroxymethyl)-aminomethan (Tris) $\mathrm{pH} 8.0$ was used, and the anti-PSMA aptamer was conjugated in $20 \mathrm{mM} \mathrm{N}$-2-Hydroxyethylpiperazine-N'-2 ethanesulphonic acid (HEPES) pH 7.4. Laser generation of AuNPs was performed utilizing a Spitfire Pro femtosecond laser system (Spectra-Physics) providing $120 \mathrm{fs}$ laser pulses at a wavelength of $800 \mathrm{~nm} .5 \times 5 \mathrm{~mm}$ gold foils were placed in the wells of a 24 well plate filled with $500 \mu \mathrm{l}$ of aptamer solution in the respective buffer. Ablation was performed while moving the plate at a constant speed of $60 \mathrm{~mm} \times \mathrm{min}-1$ in a spiral (outer radius: $3 \mathrm{~mm}$, inner radius: $1.5 \mathrm{~mm}$ ), using an axis system. Recently, we have optimized the laser parameters for laser-ablation-based generation of DNA-conjugated AuNPs[19]. Here, laser fluence was optimized in regard to maximal productivity, while avoiding degradation of the oligonucleotide. In the present study, the optimized parameters were chosen, the pulse energy was fixed at $100 \mu \mathrm{J}$, and the repetition rate was $5 \mathrm{kHz}$. In order to avoid heat-induced degradation of the aptamer, the focus position was adjusted to be $2 \mathrm{~mm}$ beneath the focus position determined in air[19].

\section{Post-generation processing of the aptamer-conjugated AuNPs}

After laser ablation, the conjugates were allowed to age overnight at $4{ }^{\circ} \mathrm{C}$ before $\mathrm{NaCl}$ was added in increments of $25 \mathrm{mM}$ by addition of $2 \mathrm{M} \mathrm{NaCl}$ in Tris-Cl or HEPES respectively. After each $\mathrm{NaCl}$ addition, the colloidal solution was mixed and incubated for $1 \mathrm{~h}$ at room temperature. The addition of $\mathrm{MgCl}_{2}$ and $\mathrm{CaCl}_{2}$ was performed after another overnight incubation at $4^{\circ} \mathrm{C}$, by addition of $1 \mathrm{M} \mathrm{MgCl}_{2}$ and $1 \mathrm{M} \mathrm{CaCl}_{2}$. Final buffer compositions were the following: miniStrep: $150 \mathrm{mM}$ $\mathrm{NaCl}, 10 \mathrm{mM} \mathrm{MgCl} 2,50 \mathrm{mM}$ Tris-Cl pH 8.0; antiPSMA: $150 \mathrm{mM} \mathrm{NaCl}, 1 \mathrm{mM} \mathrm{MgCl} 2,1 \mathrm{mM} \mathrm{CaCl}_{2}$, 0.05\% Tween 20, $20 \mathrm{mM}$ HEPES pH 7.4.

To remove the free aptamer, the ablation medium was centrifuged for $15 \mathrm{~min}$ at $15000 \mathrm{rpm}$. The supernatant was transferred into a new centrifugal tube and centrifuged for another $30 \mathrm{~min}$. The supernatant was discarded, and the pellets were pooled and resuspended in the respective buffer. This process was repeated 4 times.

\section{Characterization methods}

UV/VIS spectra of the AuNP solutions were recorded using a Shimadzu 1650 spectrophotometer. In order to determine the AuNP concentration, the absorption at $380 \mathrm{~nm}$ (mainly corresponding to the interband transition of gold) was measured. Intensities were converted 
to AuNP mass concentrations by interpolation from a linear standard calibration curve $\left(R^{2}=0.99\right)$. Standard curves were prepared with known concentrations of AuNP produced by weighing a gold target three times before and after ablation.

Transmission electron micrographs (TEM) were commissioned at Stiftung Tierärztliche Hochschule, Institut für Pathologie (Prof. Dr. W. Baumgärtner, Kerstin Rohn), and were obtained by utilizing a TEM Philip CM30 with a $0.23 \mathrm{~nm}$ resolution. One drop of the colloidal solution was placed on a carbon-coated, formvarcovered copper grid, and then dried at room temperature. Given diameters were averaged for at least 200 AuNPs. Dynamic light scattering (DLS) measurements were performed, using a Zetasizer ZS (Malvern). Three consecutive measurements were carried out and average values are presented.

The amount of aptamer bound per nanoparticle was determined by measuring the concentration of the unbound aptamer. Aptamer-conjugated AuNPs were removed by ultracentrifugation (Beckman Coulter Optima Max, $30000 \times \mathrm{g}$ ), and the adsorption of the supernatant was measured at $260 \mathrm{~nm}$ against a serial dilution of aptamer in Tris buffer. Mean values of three measurements are presented.

\section{Determination of miniStrep aptamer functionality}

The agglomeration-based streptavidin assay was performed by incubating a fixed amount of miniStrep-conjugated AuNPs (0.69 $\mathrm{nM})$ with varying concentrations of streptavidin $(0-15.9 \mathrm{nM})$ for $16 \mathrm{~h}$ at room temperature. UV/VIS was measured to monitor the shift of $\mathrm{SPR}_{\mathrm{Max}}$.

Furthermore, the aptamer activity was determined in a "golden blot"[40] format similar to the method published by Wang et al[11]. In brief, streptavidin $(0.5 \mu \mathrm{l}, 1$ $\mathrm{mg} / \mathrm{ml}$ in PBS) was spotted in 10 replicates onto a nitrocellulose membrane (Sartorius, Goettingen, Germany). After $1 \mathrm{~h}$ incubation at room temperature, blocking of the membrane was performed with $1 \%$ BSA in miniStrep selection buffer. The membrane was washed in the same buffer and incubated with a solution of AuNPs $(20 \mu \mathrm{g} / \mathrm{ml})$ for $2 \mathrm{~h}$. Finally, the membrane was washed with miniStrep selection buffer. As a negative control, BSA $(0.5 \mu \mathrm{l}, 1 \mathrm{mg} / \mathrm{ml}$ in PBS) was spotted on the membrane. Furthermore, the experiment was repeated with "bare" AuNP produced in Tris buffer in the absence of aptamer. In this experiment, the miniStrep selection buffer was replaced by $50 \mathrm{mM}$ Tris- $\mathrm{Cl} \mathrm{pH} 8.0$, in order to maintain colloidal stability of the non-stabilized nanoparticles. Environmental scanning electron microscopy (ESEM) of the membrane after incubation with AuNPs was performed with a Quanta 400 F (FEI, Eindhoven, Netherlands) in low vacuum conditions. A piece of membrane was placed on an aluminum holder and visualized without previous sputtering.

In order to determine the activity of the miniStrep aptamer bound to the AuNP surface, the conjugate $(28.5 \mu \mathrm{g} / \mathrm{ml}, 0.15 \mathrm{nM})$ was incubated with Cy3-labeled streptavidin $(166.7 \mu \mathrm{g} / \mathrm{ml}, 2.8 \mu \mathrm{M})$ for $16 \mathrm{~h}$ at room temperature, in the dark. The conjugates and bound streptavidin were removed by ultracentrifugation. The amount of streptavidin bound to the nanoparticles was determined by measuring the streptavidin concentration remaining in the supernatant, utilizing a Fluoroskan ascent fluorescence plate reader (Ex: $544 \mathrm{~nm}, \mathrm{Em}: 590$ $\mathrm{nm})$. Mean values of 4 measurements are presented.

\section{Determination of anti-PSMA aptamer functionality}

The activity of anti-PSMA aptamers conjugated to AuNPs was investigated, using a tissue microarray consisting of paraffin-embedded prostate cancer tissues (US Biomax, Rockville, MD, USA). After baking the slides at $60^{\circ} \mathrm{C}$ for $30 \mathrm{~min}$, paraffin was removed using two washing steps in xylene (10 min each). The tissue arrays were rehydrated by consecutive washes in 100\%, 95\% and $70 \%$ ethanol, followed by a washing step in $\mathrm{ddH}_{2} \mathrm{O}$ ( $5 \mathrm{~min}$ each). Antigen retrieval was performed by placing the slides in $0.01 \mathrm{M}$ sodium citrate $\mathrm{pH} 6.0$ for $15 \mathrm{~min}$ at $95^{\circ} \mathrm{C}$. Consequently slides were washed with anti-PSMA aptamer selection buffer, and blocked in 5\% goat serum (Millipore) in the same buffer. The antiPSMA selection buffer was used for all consequent assay steps. Incubation with the aptamer-modified AuNPs $(20 \mu \mathrm{g} / \mathrm{ml})$ was performed for $2 \mathrm{~h}$ at $20^{\circ} \mathrm{C}$ and $300 \mathrm{rpm}$ in an Eppendorf shaker equipped with a slide adaptor, after placing a secure seal incubation chamber (Grace Biolabs, Bend, OR, USA) filled with $800 \mu \mathrm{l}$ of the respective AuNP solution on the slide. Slides were washed two times for $5 \mathrm{~min}$ with $1 \%$ goat serum, and fixed for 15 min with $2.5 \%$ glutaraldehyde solution. Silver enhancement was performed using a silver enhancer kit (Sigma), according to the instructions provided by the manufacturer.

AuNPs conjugated with miniStrep Aptamer in HEPES buffer were chosen as a negative control. All washing and incubation steps were performed as described above. As a positive control, a rabbit anti-PSMA antibody directed against the C-terminal domain of human PSMA (Millipore) was used[41]. Here, all washing and incubation steps were performed using PBS. After incubation with $2.5 \mu \mathrm{g} / \mathrm{ml}$ rabbit anti-PSMA for $2 \mathrm{~h}$, the slides were washed two times for $5 \mathrm{~min}$ with $1 \%$ goat serum, and consequently incubated with a 1:20 dilution of $12 \mathrm{~nm}$ colloidal gold conjugated with goat anti-rabbit IgG (Jackson Immuno Research; OD at $520 \mathrm{~nm}$ of stock solution: 2) for $1.5 \mathrm{~h}$. High background of developed tissue arrays was removed as described by Springall et al [42]. 


\section{Acknowledgements}

This work was funded by the German Research Foundation Society DFG within the Excellence Cluster REBIRTH (From Regenerative Biology to Reconstructive Therapy). The authors thank Prof. C. Urbanke, PD U. Curth and Frank Hartmann (Medizinische Hochschule Hannover) for the possibility to use the ultracentrifugation facilities.

\section{Author details}

'Institut für Technische Chemie, Leibniz Universität Hannover, Callinstrasse 3, 30167 Hannover, Germany. ${ }^{2}$ Laser Zentrum Hannover, Hollerithallee 8, 30419 Hannover, Germany.

\section{Authors' contributions}

JGW and SP carried out the in situ conjugations and partial drafting of the manuscript. JGW carried out the determination of aptamer functionality. JGW and FS carried out the tissue microarray experiments. SB carried out the principal study design, manuscript drafting and supervision of nanoparticle generation. TS participated in the conception design and supervised aptamer-related work. All authors read and approved the final manuscript.

\section{Competing interests}

The authors declare that they have no competing interests.

Received: 30 March 2010 Accepted: 23 August 2010

Published: 23 August 2010

\section{References}

1. Alivisatos AP, Johnsson KP, Peng X, Wilson TE, Loweth CJ, Bruchez MP Jr. Schultz PG: Organization of 'nanocrystal molecules' using DNA. Nature 1996, 382:609-611.

2. Mirkin CA, Letsinger RL, Mucic RC, Storhoff JJ: A DNA-based method for rationally assembling nanoparticles into macroscopic materials. Nature 1996, 382:607-609.

3. Sato K, Hosokawa K, Maeda M: Colorimetric biosensors based on DNAnanoparticle conjugates. Anal Sci 2007, 23:17-20.

4. Pavlov V, Xiao Y, Shlyahovsky B, Willner I: Aptamer-functionalized Au nanoparticles for the amplified optical detection of thrombin. J Am Chem Soc 2004, 126:11768-11769.

5. Huang CC, Huang YF, Cao Z, Tan W, Chang HT: Aptamer-modified gold nanoparticles for colorimetric determination of platelet-derived growth factors and their receptors. Anal Chem 2005, 77:5735-5741.

6. Tuerk C, Gold L: Systematic evolution of ligands by exponential enrichment: RNA ligands to bacteriophage T4 DNA polymerase. Science 1990, 249:505-510

7. Ellington AD, Szostak JW: In vitro selection of RNA molecules that bind specific ligands. Nature 1990, 346:818-822.

8. Robertson DL, Joyce GF: Selection in vitro of an RNA enzyme that specifically cleaves single-stranded DNA. Nature 1990, 344:467-468,

9. Xu H, Mao X, Zeng Q, Wang S, Kawde AN, Liu G: Aptamer-functionalized gold nanoparticles as probes in a dry-reagent strip biosensor for protein analysis. Anal Chem 2009, 81:669-675.

10. Zhao W, Chiuman W, Lam JC, McManus SA, Chen W, Cui Y, Pelton R, Brook MA, Li Y: DNA aptamer folding on gold nanoparticles: from colloid chemistry to biosensors. J Am Chem Soc 2008, 130:3610-3618.

11. Wang Y, Li D, Ren W, Liu Z, Dong S, Wang E: Ultrasensitive colorimetric detection of protein by aptamer-Au nanoparticles conjugates based on a dot-blot assay. Chem Commun (Camb) 2008, 2520-2522.

12. Medley CD, Smith JE, Tang Z, Wu Y, Bamrungsap S, Tan W: Gold nanoparticle-based colorimetric assay for the direct detection of cancerous cells. Anal Chem 2008, 80:1067-1072.

13. Huang YF, Sefah K, Bamrungsap S, Chang HT, Tan W: Selective photothermal therapy for mixed cancer cells using aptamer-conjugated nanorods. Langmuir 2008, 24:11860-11865.

14. Besner S, Kabashin A, Winnik F, Meunier M: Ultrafast laser based "green" synthesis of non-toxic nanoparticles in aqueous solutions. Applied Physics A 2008, 93:955-959.

15. Connor EE, Mwamuka J, Gole A, Murphy CJ, Wyatt MD: Gold nanoparticles are taken up by human cells but do not cause acute cytotoxicity. Small 2005, 1:325-327.
16. Sylvestre J, Kabashin A, Sacher E, Meunier M: Femtosecond laser ablation of gold in water: influence of the laser-produced plasma on the nanoparticle size distribution. Applied Physics A 2005, 80:753-758.

17. Barcikowski S, Hahn A, Kabashin A, Chichkov B: Properties of nanoparticles generated during femtosecond laser machining in air and water. Applied Physics A 2007, 87:47-55.

18. Mafune F, Kohno J, Takeda Y, Kondow T, Sawabe H: Formation of gold nanoparticles by laser ablation in aqueous solution of surfactant. J PHYS CHEM B 2001, 105:5114-5120.

19. Petersen S, Barcikowski S: In Situ Bioconjugation: Single Step Approach to Tailored Nanoparticle-Bioconjugates by Ultrashort Pulsed Laser Ablation. ADV FUNCT MATER 2009, 19:1167-1172.

20. Petersen S, Jakobi J, Barcikowski S: In situ bioconjugation-Novel laser based approach to pure nanoparticle-conjugates. APPL SURF SCI 2009, 255:5435-5438

21. Sylvestre JP, Kabashin AV, Sacher E, Meunier M, Luong JH: Stabilization and size control of gold nanoparticles during laser ablation in aqueous cyclodextrins. J Am Chem Soc 2004, 126:7176-7177.

22. Petersen S, Barcikowski S: Conjugation Efficiency of Laser-Based Bioconjugation of Gold Nanoparticles with Nucleic Acids. J Phys Chem C 2009, 113:19830-19835.

23. Giljohann DA, Seferos DS, Patel PC, Millstone JE, Rosi NL, Mirkin CA: Oligonucleotide loading determines cellular uptake of DNA-modified gold nanoparticles. Nano Lett 2007, 7:3818-3821.

24. Rosi NL, Giljohann DA, Thaxton CS, Lytton-Jean AK, Han MS, Mirkin CA: Oligonucleotide-modified gold nanoparticles for intracellular gene regulation. Science 2006, 312:1027-1030

25. Massich MD, Giljohann DA, Seferos DS, Ludlow LE, Horvath CM, Mirkin CA: Regulating immune response using polyvalent nucleic acid-gold nanoparticle conjugates. Mol Pharm 2009, 6:1934-1940.

26. Bittker JA, Le BV, Liu DR: Nucleic acid evolution and minimization by nonhomologous random recombination. Nat Biotechnol 2002, 20:1024-1029.

27. Walter JG, Kokpinar O, Friehs K, Stahl F, Scheper T: Systematic investigation of optimal aptamer immobilization for protein-microarray applications. Anal Chem 2008, 80:7372-7378.

28. Kimura-Suda H, Petrovykh DY, Tarlov MJ, Whitman L: Base-dependent competitive adsorption of single-stranded DNA on gold. J Am Chem Soc 2003, 125:9014-9015.

29. Lupold SE, Hicke BJ, Lin Y, Coffey DS: Identification and characterization of nuclease-stabilized RNA molecules that bind human prostate cancer cells via the prostate-specific membrane antigen. Cancer Res 2002, 62:4029-4033.

30. Hurst SJ, Lytton-Jean AK, Mirkin CA: Maximizing DNA loading on a range of gold nanoparticle sizes. Anal Chem 2006, 78:8313-8318.

31. Zhang F, Skoda MW, Jacobs RM, Zorn S, Martin RA, Martin CM, Clark GF, Goerigk G, Schreiber F: Gold nanoparticles decorated with oligo(ethylene glycol) thiols: protein resistance and colloidal stability. J Phys Chem A 2007, 111:12229-12237.

32. Dougan JA, Karlsson C, Smith WE, Graham D: Enhanced oligonucleotidenanoparticle conjugate stability using thioctic acid modified oligonucleotides. Nucleic Acids Res 2007, 35:3668-3675.

33. Balamurugan S, Obubuafo A, Soper SA, Spivak DA: Surface immobilization methods for aptamer diagnostic applications. Anal Bioanal Chem 2008, 390:1009-1021

34. Park S, Brown K, Hamad-Schifferli K: Changes in oligonucleotide conformation on nanoparticle surfaces by modification with mercaptohexanol. NANO LETT 2004, 4:1925-1929.

35. Demers LM, Mirkin CA, Mucic RC, Reynolds RA, Letsinger RL, Elghanian R, Viswanadham G: A fluorescence-based method for determining the surface coverage and hybridization efficiency of thiol-capped oligonucleotides bound to gold thin films and nanoparticles. Anal Chem 2000, 72:5535-5541.

36. Jain $\mathrm{P}$, Huang $\mathrm{W}$, El-Sayed $\mathrm{M}$ : On the universal scaling behavior of the distance decay of plasmon coupling in metal nanoparticle pairs: A plasmon ruler equation. NANO LETT 2007, 7:2080-2088.

37. Kinoshita Y, Kuratsukuri K, Landas S, Imaida K, Rovito PM Jr, Wang CY, Haas GP: Expression of prostate-specific membrane antigen in normal and malignant human tissues. World J Surg 2006, 30:628-636.

38. Chang SS, Reuter VE, Heston WD, Bander NH, Grauer LS, Gaudin PB: Five different anti-prostate-specific membrane antigen (PSMA) antibodies 
confirm PSMA expression in tumor-associated neovasculature. Cancer Res 1999, 59:3192-3198.

39. Gong MC, Chang SS, Sadelain M, Bander NH, Heston WD: Prostate-specific membrane antigen (PSMA)-specific monoclonal antibodies in the treatment of prostate and other cancers. Cancer Metastasis Rev 1999, 18:483-490.

40. Brada D, Roth J: "Golden blot"-detection of polyclonal and monoclonal antibodies bound to antigens on nitrocellulose by protein A-gold complexes. Anal Biochem 1984, 142:79-83.

41. Murphy GP, Greene TG, Tino WT, Boynton AL, Holmes EH: Isolation and characterization of monoclonal antibodies specific for the extracellular domain of prostate specific membrane antigen. J Urol 1998, 160:2396-2401.

42. Springall DR, Hacker GW, Grimelius L, Polak JM: The potential of the immunogold-silver staining method for paraffin sections. Histochemistry 1984, 81:603-608.

doi:10.1186/1477-3155-8-21

Cite this article as: Walter et al:: Laser ablation-based one-step

generation and bio-functionalization of gold nanoparticles conjugated with aptamers. Journal of Nanobiotechnology 2010 8:21.

\section{Submit your next manuscript to BioMed Central} and take full advantage of:

- Convenient online submission

- Thorough peer review

- No space constraints or color figure charges

- Immediate publication on acceptance

- Inclusion in PubMed, CAS, Scopus and Google Scholar

- Research which is freely available for redistribution

Submit your manuscript at www.biomedcentral.com/submit 